\title{
Cons(des)trucción del espacio urbano y el discurso identitario en la obra de Antonio José Ponte
}

El presente artículo estudia cómo emplea Antonio José Ponte la idea de espacio urbano para construir a partir de esta el discurso identitario cubano. El paisaje urbano de La Habana, en general, y su decaimiento, en especial, sirven como punto de partida para una crítica aguda de la postura ideológica del gobierno comunista de Cuba que Ponte desarrolla en su ensayo "Un arte de hacer ruinas," su novela Villa Marista en plata y la película La Habana - Arte nuevo de hacer ruinas. El planteamiento pontiano del impacto destructivo del sistema socialista en el espacio urbano, más notablemente en lo que concierne al llamado "problema de la vivienda", revela unas coincidencias casi literales en los puntos claves con la visión de Mijaíl Bulgakov expresada de manera más contundente en su icónica obra El Maestro y Margarita. A pesar de que los dos autores y las respectivas realidades desde las cuales, y sobre las cuales, han escrito están separadas por más de ocho décadas, el modelo soviético de desarrollo urbano determina en buena medida los problemas que sufrió Cuba durante el Periodo Especial y más allá.

\footnotetext{
Bueno -respondió aquel pensativo-, son hombres como todos... Les gusta el dinero pero eso ha sucedido siempre... A la humanidad le ha gustado siempre el dinero, sin importarle de qué estuviera hecho: de cuero, de papel, de bronce o de oro. Bueno, son frívolos..., pero iy qué?..., también la misericordia pasa a veces por sus corazones... Hombres corrientes, recuerdan a los de antes solo que a éstos les ha estropeado el problema de la vivienda...

Mijaíl Bulgakov, El Maestro y Margarita
}

La idea de que existe cierta relación entre la configuración del espacio urbano y el formato socioeconómico del país, desarrollada más notablemente en los trabajos de David Harvey, da un giro inesperado en la producción literaria de Antonio José Ponte. Según propone Harvey: 
We can ... argue that objective conceptions of time and space are necessarily created through material practices and processes which serve to reproduce social life... The objectivity of time and space is given in each case by the material practices of social reproduction, and to the degree that these latter vary geographically and historically, so we find that social time and social space are differentially constructed. Each distinctive mode of production or social formation will, in short, embody a distinctive bundle of time and space practices and concepts. ("Condition of Postmodernity" 204)

De este planteamiento se deduce que el aspecto cualitativo del espacio se extiende y afecta a la distribución de objetos en las áreas urbanas, tal y como señala Harvey, en particular en su charla "The Urbanization of AntiCapitalist Struggle". Sin embargo, para Ponte es más bien el estado en el que se encuentran estos objetos. La construcción y crecimiento de las urbes en la sociedad capitalista que estudia Harvey se convierte en ruina y decaimiento en La Habana socialista de Ponte. Por otro lado, también da inicio a un tipo único de crecimiento en el cual importa la dirección hacia dónde dicho crecimiento se realiza. A partir de estas premisas, me interesa determinar cómo la especificidad de la organización de los espacios urbanos impacta el discurso nacional cubano y qué rol juega el factor sociopolítico e ideológico en esta definición. Mi objetivo, por lo tanto, es examinar el papel del concepto de espacio urbano, en general, y del espacio arruinado o deteriorado en la construcción del discurso nacional cubano, así como en el discurso contestatario de dicha ideología en la poética narrativa de Ponte.

Independientemente del país de origen, muchas literaturas - o por lo menos algunos autores que las representan - han tratado de definir o redefinir el discurso nacional a partir de la geografía, el paisaje y sus rasgos distintivos. Según afirman Elfie Rembold y Peter Carrier, "narratives of nations, nationhood and identities are not simply products of the imagination, they require a natural or 'organic' rootedness" $(362){ }^{\mathrm{I}}$ Para Ponte, junto con el concepto de los alimentos que utiliza en su ensayo "Las comidas profundas", la noción del espacio urbano es un constituyente muy importante de la identidad nacional. Según afirma el autor en una de sus entrevistas, la comida y las ruinas, dos motivos recurrentes de sus escritos, "son metáforas de Cuba", aunque admite que entre las dos, le da preferencia a las ruinas (Serna y Solana I3I). En el ensayo "Un arte de hacer ruinas", a partir del concepto de espacio, Ponte construye el discurso nacional basado en una fuerte carga ideológica. La extensión muy limitada de la isla es para él uno de los factores que afectan a la identidad nacional. La condición isleña de Cuba posee implicaciones políticas e ideológicas y se 
presta para ciertas conclusiones en cuanto a los rasgos determinantes del discurso nacional.

Mientras que la mayoría de los países del mundo en la época de la globalización han experimentado el fenómeno llamado "desterritorialización", introducido en los trabajos de Gilles Deleuze y Félix Guattari y desarrollado por Arjun Appadurai, el impacto de la globalización en la Cuba socialista, con el aislamiento político y económico inicial y la posterior reinserción en la economía mundial, ha obtenido resultados mixtos, según plantea Pedro Monreal González. Por un lado, Cuba dispone de una fuerza laboral relativamente cualificada y de unas infraestructuras básicas satisfactorias. Por otro, las políticas actuales de desarrollo impiden el uso efectivo de dichos recursos para acelerar la adhesión de la isla a los procesos globales (Monreal González 52; 69). Teniendo en cuenta esta observación y con la debida consideración del carácter único del caso cubano, propongo que las experiencias cubanas están ligadas a la noción de sitio y de lo local quizás más que en otras parte del mundo. Por otro lado, las experiencias locales son recibidas de forma distinta, tanto por los cubanos de diferentes posturas ideológicas, como por los de la diáspora. El factor de la emigración numerosa de una isla pequeña, en medio del aislamiento global, produce unas conexiones que sustituyen la falta de contactos oficiales con el resto del mundo. En relación a este fenómeno, muchos autores ven la diáspora con sus respectivos hábitats como ciudades paralelas o gemelas de La Habana: “... for Havana the emigration of a good part of its former inhabitants also signified the beginning of a relationship with its 'mirror city,' Miami, where, ninety miles from the coast, those who left began to re-create and re-found the "lost city"' (Bobes 20). Algunos llegan incluso a establecer una red de "pancubanidad": "... in Cuba the limits of the cubanía were being rethought and the idea of a common culture of 'Greater Cuba,' with hubs not only in Havana and Miami but also in other European, and U.S. cities, was being articulated" (Birkenmaier y Whitfield 6). Todos estos factores hacen del espacio cubano, más específicamente, habanero, un campo fértil para la búsqueda de una identidad nacional.

Las características del espacio en la obra de Ponte se refractan por el problema de la crisis de la vivienda que, en el contexto cubano, adquiere naturaleza no solo socioeconómica, sino también política. Las primeras menciones de la crisis de la vivienda se remontan a la Europa de finales del siglo XIX y principios del XX (Del Real y Scarpaci 55). En la modernidad, el problema se sigue agudizando debido al flujo creciente de los migrantes provincianos a las áreas urbanas y al déficit de acceso a vivienda para las personas de bajos ingresos. 
A pesar de que el déficit de vivienda se ha convertido en un problema global, la situación de los países socialistas posee rasgos distintivos que conviene destacar. Uno de ellos tiene que ver con el rol del estado en la solución del dicho problema. A diferencia de las economías capitalistas en las cuales la adquisición de la vivienda ha sido de forma predominante responsabilidad del ciudadano, en los países socialistas el gobierno se involucra más activamente ofreciendo al ciudadano varias soluciones que se han puesto en práctica con diferentes grados de éxito. El problema más común de este modus operandi es la imposibilidad de satisfacer las necesidades crecientes de la población, lo cual provoca, a su vez, una serie de consecuencias negativas como los largos tiempos de espera, la baja calidad de la construcción y la reducción de los espacios habitables. Por otra parte, en las sociedades socialistas es prácticamente desconocido el fenómeno de las barriadas o favelas, que son los signos distintivos de los países capitalistas de Latinoamérica (Delgado). Dicha ausencia, sin embargo, no es vista como positiva por los autores que han investigado el problema. Según Babar Mumtaz, el crecimiento de las ciudades en forma de barriadas es un signo de desarrollo y progreso (cit. en Lawless 202). Como nota Damaris Puñales-Alpízar,

La Habana ha vivido, en los últimos años, una inmovilidad compleja. Aunque el número de inmigrantes de otras provincias y ciudades ha seguido creciendo, la oferta de vivienda se ha mantenido bastante inmóvil. Para resolver el problema básico de dónde vivir, los nuevos habitantes de la ciudad no han fundado ciudadelas o barrios marginales en las orillas, no han construido casas de cartón y cinc en las afueras, tampoco han tenido la opción de adquirir una vivienda en el mercado de bienes raíces - inexistente en Cuba - sino han comenzado a fragmentar edificios antiguos residenciales.

Estas observaciones indican que el modo de vida socialista no excluye la precariedad de las condiciones de vida sino que desarrolla otras formas de vivienda precaria, como veremos más adelante.

Para entender mejor el trasfondo de la crisis cubana, habría que ver cómo se desenvolvió dicha cuestión en la Unión Soviética, ya que constituyó el prototipo socioeconómico para Cuba. La crisis de la vivienda en una sociedad socialista se inició por primera vez en la Rusia posrevolucionaria después del año I9I7, se agudizó hacia los años I930, y con medidas gubernamentales más o menos exitosas, duró hasta la desintegración del país en el año i991. Al principio se resolvió en parte con la expropiación, modificación y división de la vivienda de la clase media y alta. Los "kommunalki" (vivienda comunal) resultantes de este proceso 
lograron acomodar a muchas personas de los sectores desaventajados. Como observa Orlando Figes,

the policy had a strong ideological appeal, not just as a war on privilege, which is how it was presented in the propaganda of the new regime ..., but also as part of a crusade to engineer a more collective way of life. By forcing people to share communal apartments, the Bolsheviks believed that they could make them communistic in their basic thinking and behaviour. Private space and property would disappear, the individual ("burgeouis") family would be replaced by communistic fraternity and organization, and the life of the individual would become immersed in the community. (9)

Al mismo tiempo, estos experimentos sociales produjeron tensiones que tenían que ver principalmente con la prioridad de la vivienda y múltiples casos de corrupción, nepotismo, y abusos de poder en su distribución. La novela de Mijaíl Bulgakov, El Maestro y Margarita, un fragmento de la cual he elegido como epígrafe, y a la que voy a referirme más adelante, construye la mayor parte de su narrativa alrededor de este tema. El tipo de multiplicación de los espacios en los "kommunalki", criticado por Bulgakov, se acerca considerablemente al planteamiento de Ponte en torno al urbanismo de La Habana.

Después de la Segunda Guerra Mundial, cuando un porcentaje muy alto de la infraestructura del país había sido destruido, la necesidad de alojamiento condujo al plan estatal de construcción de viviendas económicas y rápidas de edificar. El surgimiento de los "hrushevki", unos edificios de departamentos básicos, con un metraje sustancialmente reducido y solo los elementos indispensables de la infraestructura, señala un nuevo periodo en la cuestión de la vivienda. Como señala Matveev, la construcción de alojamientos de nuevo tipo se convirtió en campo de contienda ideológica y de afirmación de los valores socialistas por parte del discurso político oficial. El nuevo tipo de vivienda se oponía, por un lado, al modo de vida capitalista y, por otro, desafiaba la desigualdad en las condiciones de vida que estaba muy presente en la época de Stalin. Por otra parte, la implementación de los recortes severos de las dimensiones de una vivienda típica se entendía de acuerdo a la ideología oficial que profesaba que en un país socialista el ciudadano no necesitaba lujos ni excesos (Matveev 254). Esta línea ideológica enfatiza la contraposición fundamental de lo antiguo y lo nuevo, según lo demuestra, por ejemplo, la directiva del Comité Central del Partido Comunista adoptada en 1955 que representa el tipo ideal de vivienda soviética (Matveev 255). Un ejemplo muy significativo y sintomático, tanto de la época como de la ideología 
comunista, fue la reducción de la cocina considerada un elemento innecesario en el modo de vida comunista donde dominaría el sistema comunitario de alimentación (Matveev 256). Reflexionando sobre la repercusión de esta tendencia en la Cuba socialista, Velia Bobes describe la transformación de La Habana en términos similares, notando que "the great city of the past, represented as the incarnation of sin and of the excesses of pleasure, perversion, and inequality, gives way to the Sovieticized city of the end of the I970s and the I980s..." (23). Enfatiza, asimismo, la reducción de los espacios en las nuevas construcciones y la uniformidad, ejemplificada en el barrio Alamar (Bobes 23). Para Bobes,

Soviet Havana, with Alamar as its paradigmatic emblem, began to approach the principle of uniformity as the ideal of the city (constructive, spatial, and social), and the imaginary was articulated around the negation of social diversity via the representation of the social subject - the new man - as its resident. (23)

La simplificación de la construcción y la uniformidad conducen a la disminución de la importancia del trabajo de los arquitectos. Incluso los constructores profesionales son complementados por las llamadas "microbrigadas" - futuros habitantes de los edificios sin conocimiento de construcción (Bobes 23). Como testifica Mario Coyula,

a combination of anonymous egualitarianism, technocratic bureaucracy, and dogmatism in people who deemed themselves to be repositories of absolute truth imposed rigid models copied from other climatic and cultural contexts, undermined the authority and image of the architect as creator... . (37)

Como culminación de este proceso, en 1967 se disolvió la Asociación de Arquitectos que fue sustituida por el Centro Superior de Construcción (Coyula 44).

Este breve recuento demuestra que el problema de la vivienda en la sociedad socialista lleva una carga ideológica significativa, cuyo propósito es desafiar el estilo de vida capitalista al tiempo que mantiene bajo control a sus propios ciudadanos. Dado su aislamiento internacional y su precaria situación económica, Cuba también necesitaba un fuerte discurso ideológico para mantener el descontento bajo control. Si bien la tendencia a acusar al imperialismo mundial de todos los problemas domésticos ha estado presente en la propaganda oficial de Cuba como lo estaba en la URSS, Ponte destaca otra táctica del aparato ideológico oficialista.

A pesar de que las crisis de la vivienda en la URSS y en Cuba comparten muchos rasgos - como la multiplicación de ambientes dentro de 
un espacio limitado o el problema de la división entre lo privado y lo público - Ponte también señala problemas que son característicos de la Cuba socialista. Los más destacados son la ruina de los edificios y la proliferación de espacios vacíos. Los dos contribuyen a la formación del discurso ideológico en relación con el espacio urbano y, por lo tanto, merecen atención.

La multiplicación de espacios conocida en las prácticas sociales de la URSS con el nombre de "kommunalki" se transforma en la realidad cubana en el fenómeno de las llamadas "barbacoas", que poseen un correlato implícito con la vivienda precaria de otros países de Latinoamérica (barriadas, favelas), como muestran Patricio Del Real y Joseph Scarpaci (69). Pese a la idea inicial que pueda sugerirle el término al lector que no esté familiarizado con la realidad cubana,

[u]na "barbacoa" en la Habana no es una parrilla donde puedes asar un poco de carne, unos buenos chorizos, y darte un banquete de muerte. Una "barbacoa" en La Habana es una división hecha de madera aprovechando que muchos de los pilares de las casas coloniales eran altos. A la altura mínima para no caminar agachado, se colocan unas vigas de madera que hacían una especie de segundo piso que una vez forrado de madera o incluso con una pequeña placa de concreto, duplicaba la superficie habitable intentando solucionar el crítico y eterno problema de la vivienda en Cuba. Una pequeña escalera te conduce a ese segundo habitáculo, que en lo único que se parece a una "barbacoa" es en el tremendo calor que pasas cuando estás durmiendo allá arriba. Si no tienes un ventilador a todo meter, "te asas," y con el ventilador, "te cocinas". (Aida) ${ }^{2}$

Detrás del irónico nombre existe, sin embargo, un problema serio de crecimiento desproporcionado de las áreas urbanas. A diferencia de las barriadas en países en vías de desarrollo como Colombia, Perú o Brasil, donde la llegada de nuevos residentes contribuye a la expansión de los límites de la ciudad existente, el crecimiento en la Cuba socialista es literalmente hacia adentro, sin que cambien los límites de la urbe. Este fenómeno se puede comparar con el concepto de "la quinta dimensión" que Bulgakov utiliza con ironía en su novela:

Quien conozca bien la quinta dimensión puede ampliar cualquier local todo lo que quiera y sin ningún esfuerzo, y además, le diré, estimada señora, que hasta unos límites incalculables. Yo, personalmente - siguió Koróviev -, he conocido a gente que no tenía ni la menor idea sobre la quinta dimensión, ni sobre nada, y que hacía verdaderos milagros en eso de agrandar sus viviendas. Por ejemplo, me han hablado de un ciudadano que recibió un piso de tres habitaciones $\mathrm{y}$, sin conocer la 
quinta dimensión ni demás trucos, la convirtió en un piso de cuatro, dividiendo con un tabique una de las habitaciones. Después cambió este piso por dos separados en distintos barrios de Moscú: uno de tres y otro de dos habitaciones. Convendrá usted conmigo en que ya eran cinco habitaciones. Uno de ellos lo cambió por dos pisos de dos y, como fácilmente comprenderá, se hizo dueño de seis habitaciones, aunque completamente dispersas en Moscú. Cuando se disponía a efectuar el último canje, y el más brillante, insertando un anuncio para cambiar seis habitaciones en distintos barrios por un piso de cinco, sus actividades, y por razones ajenas a su voluntad, quedaron paralizadas. Puede que ahora tenga alguna habitación, pero me atrevo a asegurar que no será en Moscú. Ya ve usted, ¡qué lagarto, y luego me habla de la quinta dimensión! (I46)

Como se ve, en la realidad soviética también se puede atestiguar el crecimiento hacia adentro. Sin embargo, en el caso cubano es, además, hacia arriba. A la pregunta del narrador de "Un arte de hacer ruinas" sobre "hacia dónde está creciendo esta ciudad," el tutor le contesta: "Hacia adentro, en barbacoas" (Ponte, "Un arte" 25).

Para reforzar el efecto (anti)estético, Ponte construye una idea gemela del crecimiento hacia arriba: el crecimiento hacia abajo. "Cuando no encuentras tierra nueva, cuando estás cercado, puede quedarte todavía un recurso: sacar a relucir la que está debajo de lo construido", dice el profesor D., "excavar, caminar en lo vertical" (Ponte, "Un arte" 33). Muy significativamente, la construcción de este último tipo de edificio se realiza con los escombros de los edificios derrumbados. Entendida de manera metafórica, esta imagen encierra varios sentidos. En primer lugar, el botón con un ancla que encuentra el narrador en la casa de su tutor representa la inmovilidad o, mejor dicho, la imposibilidad de moverse, una isla anclada por abajo como un barco. Además, frente la imposibilidad de salir de la isla, la idea de la huida se transforma en crecimiento hacia adentro. ${ }^{3}$ Como nota Cecelia Lawless, la metáfora espacial del crecimiento para adentro en los escritos de Ponte está estrechamente vinculada a la noción de la identidad nacional cubana (204). Aparte de la búsqueda de "la conexión de la isla con el continente" (Ponte, "Un arte" 33), la idea de caminar hacia abajo, dada la experiencia negativa de Ponte con la UNEAC y su expulsión en el 2003 debido a su colaboración con la revista Encuentro de la Cultura Cubana (Rodríguez 575), alude a una especie de espacio underground en el que se encuentra el intelectual cubano. Esto, a su vez, recuerda el fenómeno difundido en el contexto literario de la URSS con la expresión de "escribir para el escritorio" (o, traducido literalmente, "escribir para adentro del escritorio"). Se trata de la imposibilidad de los escritores que no pertenecían a la Unión de los Escritores Soviéticos de publicar sus obras. 
En "Un arte de hacer ruinas" de Ponte hay varias referencias a este fenómeno, como, por ejemplo, los libros clandestinos de los que el profesor D. no quiere que "se enteren en la facultad" (3I). En la película Habana Arte nuevo de hacer ruinas, Ponte es aún más explícito al destacar que el escritor en la Cuba socialista no existe si no pertenece a un sindicato oficialmente sancionado por el gobierno. Por consiguiente, la palabra de dicho escritor no tiene peso ni credibilidad en la sociedad cubana (Habana - Arte nuevo de hacer ruinas 36:5I). De hecho, el edificio gemelo que se está edificando debajo se está construyendo con las paredes de la casa del profesor D., que, al derrumbarse, lo mata. De este modo su muerte se convierte en una metáfora de la muerte profesional que experimenta un artista disidente en una sociedad autoritaria.

El problema de la multiplicación de los espacios a causa del crecimiento poblacional con su subsecuente deterioro se conoce en las obras de Ponte con el nombre de "tugurización". Según Ponte, "la tugurización es la capacidad que tiene una ciudad sobrepoblada para hacer divisiones dentro del espacio urbanizado, y de convertir esos lugares en tugurios hasta devaluarlos arquitectónicamente, de buscar nuevos espacios habitables dentro de unos límites físicos fijos" (Puñales-Alpízar). En palabras del propio autor, la tugurización constituye una "herramienta útil a la hora de historiar cómo un espacio digno llega a transformarse en un rincón de mala muerte" ("La Habana: Un paréntesis" 70). Además de a los sectores desaventajados de la población, la tugurización afecta también a los propietarios de vivienda que se ven obligados a desprenderse de la mayor parte de sus posesiones para que las autoridades puedan acomodar más gente. Es llamativa la historia de los dueños de la finca que aparece en Habana - Arte nuevo de hacer ruinas, pues en este caso la tugurización se convierte en un proceso bidireccional. Por un lado está la población que modifica la vivienda por cuenta propia para albergar más gente $y$, por el otro, el gobierno que interviene para dividir las propiedades ajenas con tal de que acomoden más personas $\mathrm{y}$, de este modo, ayuden a solucionar el tema de la vivienda.

De la multiplicación de espacios se deriva, en parte, el problema de arruinamiento. Aparte del estado insatisfactorio de las viviendas, el factor de la tugurización juega un papel central en la tendencia a modificar la estructura arquitectónica de los edificios, que lleva al arruinamiento: "...tanto intento de vivir terminaba casi siempre en lo contrario," escribe Ponte ("Un arte" 3I). La teoría detrás del fenómeno de un edificio arruinado que adapta Ponte tiene su origen en el escritor alemán Georg Simmel. En su ensayo "Las ruinas", Simmel trata el concepto de las ruinas en la cultura europea, interpretando la arquitectura como un punto frágil 
de equilibrio entre la naturaleza y el artificio, que no es duradero y termina en la ruina y el abandono. Ponte trasplanta esta idea al contexto cubano donde adquiere otro contenido y enfoque. A diferencia de las ruinas europeas, que no están habitadas y se posicionan como un fenómeno estético, según Simmel, las cubanas poseen otro sentido y simbolismo para Ponte. Lo que en el arte europeo se convirtió en el símbolo romántico de la decadencia, en La Habana de Ponte representa la degradación y, al mismo tiempo pierde su simbolismo quedando reducida a realidad repugnante. Contrario a lo que sostiene Jennifer Ruth Hosek al caracterizar las ruinas de Ponte como "allegorical, lyrical, and literary" (219), puede afirmarse que Ponte ve semejante efecto (anti)estético por el hecho de que sean unas ruinas habitadas, a diferencia de las europeas. Por lo tanto, para él la lucha entre la naturaleza y el arte se convierte en la "lucha entre tugurización y estética milagrosa" (Ponte, "Un arte" 3I). Además, son ruinas habitadas que muchas veces son declaradas inhabitables. En este sentido no solo ofrecen referencias políticas al régimen castrista, que convierte toda la isla en un sitio imposible para vivir, sino que también transforman el espacio urbano en una escena de crimen perfecto que, junto con la víctima, destruye el escenario. De este modo, se destaca el potencial destructivo de las ruinas que se convierten en asesinas que matan a sus habitantes. Este potencial destructivo, a su vez, podría interpretarse como alegoría del clima político de la isla, que es inhabitable debido a la represión de las libertades civiles, la censura y los peligros que presenta la vida en la isla.

La idea de arruinamiento y destrucción de un espacio urbano pasa después a la sociedad, sus integrantes, su modo de vida e incluso al gobierno. Ponte dirige su crítica al régimen castrista y, personalmente, a Fidel Castro: "Fidel Castro es la gran ruina de este país. No solo por lo que ha arruinado sino que él... estamos esperando a que él se desplome" (Habana - Arte nuevo de hacer ruinas 38: 49). Es de notar cómo Ponte traza la conexión entre la ruina humana, la ruina de una ciudad y la necrofilia, como propone Guillermina De Ferrari (88-89). Las reverberaciones de esta idea también las encontramos en Hosek que arguye que las ruinas, junto con sus habitantes, representan una especie de "symbiotic decrepitude" (222) que es sumamente estática y transcurre, según ella, en un solo tiempo: "In the film, time consists of one homogeneous, unchanging present and one homogeneous, unchanging past that overlap upon and organize what is depicted as one city space. Diegetic time stands still, while the plot often moves backward as each character recounts his or her tale" (223).

Hosek sostiene que Ponte, al colocar a sus personajes en un espacio sin tiempo, ignora el potencial vital y creativo de estos individuos para 
mantener la línea en contra del régimen. Dicha interpretación acerca La Habana de Ponte a la Venecia de Simmel o al San Petersburgo lotmaniano. Llamándola "unique variation on a Potiomkin village" (219), Hosek enfatiza su teatralidad, como la de Venecia o San Petersburgo. Hablando del carácter estático de Venecia, Simmel nota: "There is probably no city whose life is carried out so fully in a single tempo... Ambivalent is the character of these places: with their lack of vehicles, their narrow, symmetrical enclosure assumes the look of a room" ("Venice" 44-45). La teatralidad de San Petersburgo, según Lotman, se debe mayormente al hecho de que se distinga por un apego único al estilo que demuestran los conjuntos arquitectónicos, que no se desintegran, como en las ciudades con una historia más larga (Turoma 39). La teatralidad de La Habana, a su vez, se resalta por medio de los espacios invertidos, como señala Ponte: "Pasamos a una sala que podía ser trastienda de algún anticuario. Un sofá cama era la única concesión hecha a una casa. Se ofrecían bancos de parque en lugar de muebles, el espacio estaba subdividido por pedazos de rejas. Las lámparas eran enormes faroles de portales y en las paredes colgaban rótulos de calles ("Un arte" 29).

El comentario de Ponte sobre el estado ruinoso de La Habana que se quedó congelada en el tiempo es de naturaleza política. Dejando el factor económico al margen de su enfoque primario, Ponte mantiene que el gobierno utiliza el fenómeno de arruinamiento con fines propagandísticos. El propósito de las ruinas en La Habana, afirma él, es simular una invasión de los Estados Unidos que nunca sucedió y sus consecuencias:

... I venture that there is a perfect correspondence between a destroyed Havana and an official discourse that has always dwelled on waiting for a U.S. military invasion and on its milder corolary, the U.S. economic embargo against Cuba. Seen as such, the city in ruins constitutes the best stage for such damning exercises. Havana is the simulacrum of an invaded city, the (actual-size) model on the campaign table of the state. Hence, Havana can show its collapsed buildings and its underpinnings in the same way that a victim shows the marks of a violent act to a court. (Ponte, "La Habana” 264)

En este contexto, es significativa, asimismo, la mención de los refugios antiaéreos en "Un arte de hacer ruinas" (29). Irónicamente, estos comentarios políticos de Ponte rompen la singularidad (single tempo) del tiempo de La Habana, pues los refugios antiaéreos representan la propaganda típica de la Guerra Fría, enfocada en la prevención de la amenaza, y las ruinas vienen a ser la consecuencia de algo que ya supuestamente habría ocurrido. ${ }^{5}$ 
La idea de destrucción y aniquilación que acabo de exponer contradice la idea de proliferación. Sin embargo, a diferencia de la película Suite Habana que analiza Hosek, donde la dirección es positiva, en Ponte es negativa pues habla de la proliferación de espacios vacíos: "Donde caía una edificación no levantaban otra. Salíamos del derrumbe del modo más barato, con la construcción de un parque, de un espacio vacío. Las parejas hallaban los rincones que podían, las mujeres quedaban preñadas en aquellas citas, las salas de maternidad se repletaban" ("Un arte" 32).

Aparte de las ya destacadas tendencias contradictorias de crecimiento y arruinamiento, con la aún más paradójica conservación de los límites existentes de la urbe, el desplazamiento de las líneas divisorias entre lo propio y lo ajeno constituyen otro aspecto muy importante en la formación de la identidad cubana, según Ponte. En las sociedades socialistas este fenómeno se manifiesta en la incursión del estado en los espacios privados de los ciudadanos entendidos estos tanto literal como metafóricamente. Un comentario sarcástico de Ponte sobre este tema aparece en su obra Villa Marista en plata. Las escenas que abren el libro ofrecen una introspección sobre el rol de los espacios privados en la vida sociopolítica cubana regida por la ideología comunista. La privacidad del ciudadano es invadida por los funcionarios estatales y destruida por la instalación de las cámaras de vigilancia destinadas a espiar (pero con su consentimiento) las conversaciones subversivas que los ciudadanos puedan tener. La privacidad de la persona también se ve invadida en "Un arte de hacer ruinas", y aunque se realiza de otro modo, el rol indirecto del estado en dicha invasión sigue presente. La incapacidad del gobierno de solucionar el problema del alojamiento conduce a la eliminación casi completa de la privacidad, pues la vivienda socialista se convierte en un espacio constantemente compartido. El replanteamiento de la relación entre lo privado y lo público se produce también por el hecho de que

many of the great mansions abandoned by those who went into exile are turned into offices, schools, or lodgings for students from the countryside who are on scholarship; the old private clubs are opened to the public and become Workers' Social circles, with the most exclusive of all, the Country Club, turned into an area that houses the new National Schools of Art - open to the children of workers and farmers. (Bobes i9-20)

Vista desde esta perspectiva, la readaptación de los espacios posee, por consiguiente, un sentido no solo práctico, sino también simbólico, que conduce a la resemantización de la ciudad y, por extensión, de los derechos del ciudadano. 
La mayoría de los componentes del problema de la vivienda que he examinado hasta ahora en la obra pontiana goza también de una representación muy amplia en El Maestro y Margarita, reflejando todos los problemas del régimen socialista en cuanto al estilo autoritario y su manejo de la pobreza y desigualdad. Lo que evidentemente hace la escritura de Ponte, similar a la de Bulgakov, es suponer que el régimen, a través de cuestiones relacionadas con el modo de vida, controla la población y usa dicho control como arma ideológica. Semejante statu quo inevitablemente produce transformaciones en la relación entre los ciudadanos y el estado. Como la apoteosis del estancamiento y arruinamiento en una sociedad socialista, Ponte recurre a una imaginería semejante a la de Bulgakov. En la película Habana - Arte nuevo de hacer ruinas, Ponte afirma:

Mann mandaría acá a un esteta, un músico y estaría muriendo. Y se enamoraría de alguien aquí, de la ciudad también, y sería el ideal de la ciudad. Y podría presentarse del diablo vestido de bicitaxista. Y tendría un diálogo con ese bicitaxista, diálogo teológico. Y al final moriría mirando una ruina, o le cae encima una cornisa. 0 de una rara fiebre que cogió mirando las ruinas. Ese sería el Thomas Mann de La Habana. (ıı:o9)

Se refiere a la trama de La muerte en Venecia de Thomas Mann, pero también menciona al diablo ampliando de este modo el contexto literario y brindando intertextualidad a la película. La figura del diablo remite al Mefistófeles de Doctor Fausto. Por otro lado, el diablo de Bulgakov aparece en la Moscú de los años I930 "encubierto", presentándose como un extranjero, especialista en magia negra y en textos antiguos. También sostiene un diálogo teológico con los habitantes de Moscú y se involucra en la cuestión del déficit de vivienda. En este contexto, parece aún más impactante el hallazgo de Galina Vasilyeva, elaborado en su artículo "Kvartirnyi vopros $\mathrm{v}$ romane Bulgakova: istoriya predpolagaemogo konteksta" ("La cuestión de la vivienda en la novela de Bugakov: La historia de un supuesto contexto"; mi traducción). Después de un análisis exhaustivo de El Maestro y Margarita y las publicaciones periódicas de la época, Vasilyeva llega a la conclusión de que la novela de Bulgakov debe su imaginería y sus personajes (sobre todo el diablo llamado Woland) a dos revistas literarias contemporáneas a la creación de su novela, Satirikon y Budilnik, ambas similares a la famosa revista europea Simplicissimus de finales del siglo XIX y comienzos del XX. El emblema de Budilnik, señala Vasilyeva, era la imagen de Mefistófeles que, según la visión de los creadores de la revista debía poner en orden la publicación de las 
producciones literarias. El Mefistófeles de Budilnik tiene rasgos en común con el Woland de Bulgakov hasta tal punto que podría considerarse su prototipo literario. Según sostiene Vasilyeva, "Mefistófeles concentra en sí todas las misiones: un experto en la vida social, un oponente desafiante, un protervus de la escolástica medieval. En cierto sentido, su actividad se podría definir como artístico-literaria. El diablo es escritor y presenta el producto de su esfuerzo artístico" (I25; mi traducción).

Se crea la impresión de que Ponte y Bulgakov coinciden en un punto que está fuera (y más allá) de las ruinas de La Habana: en la figura del Mefistófeles de Thomas Mann, y por extensión, el de Goethe. Ambos escritores recurren a la figura del diablo que toma la posición de árbitro para expresar su opinión imparcial en cuanto a los problemas del sistema socialista, siendo la cuestión de vivienda y su déficit el primero de ellos, y que traducido al plano metafórico se relaciona con las restricciones de las libertades civiles y la libertad de la expresión artística. ${ }^{6}$

Tomando en consideración la analogía que traza Ponte entre Mefistófeles y la propaganda oficial del régimen castrista, su afirmación de que La Habana se ha convertido en un lugar que "es menos ciudad viva que paisaje de legitimación política" ("La Habana: Un paréntesis" 204) adquiere un nuevo sentido y como tal tiene que ser leída "in terms of power dynamics" (Rojas I28). De ahí las transformaciones que sufre el paisaje urbano - sus límites inalterables, su crecimiento anómalo, su desplazamiento de la división entre los espacios públicos y privados. Para Ponte,

Havana has been paralyzed for fifty years, and we must face that paralysis and the ruin that it has brought about. It will be necessary to determine what can be removed, to save what ought to be conserved, and to decide new projects for the city. The magnitude of the disaster is such that we might speak of a fourth founding of the city. ("La Habana" 269)

La Habana socialista presenta un panorama bastante contradictorio en cuanto a sus características geográficas y la naturaleza del espacio urbano. Por un lado, la finitud del espacio es sofocante, y la condición isleña de Cuba con su territorio limitado coincide cabalmente con las restricciones del régimen socialista. Por otro, dentro de este espacio restringido se observa una impactante proliferación de otros espacios cada vez más pequeños. El espacio urbano, definido en las sociedades capitalistas según sus infraestructuras específicas y características para este formato sociopolítico, se convierte en la Cuba comunista del Periodo especial en una infraestructura arruinada. Sin embargo, precisamente esta 
característica del espacio urbano socialista determina el modelo de vida urbana y el curso ideológico hasta tal punto que Ponte lo llega a denominar el "arte de hacer ruinas:" con esto alude tanto a la intencionalidad como al artificio y la separación de la realidad a favor de la ideología impuesta y la propaganda que facilita el control gubernamental sobre las masas.

University of Toronto

NOTAS

I Como un ejemplo clásico se podría citar al Sarmiento del Facundo: "El mal que aqueja a la República Argentina es la extensión: el desierto la rodea por todas partes y se le insinúa en las entrañas; la soledad, el despoblado sin una habitación humana, son, por lo general, los límites incuestionables entre unas y otras provincias. Allí la inmensidad por todas partes: inmensa la llanura, inmensos los bosques, inmensos los ríos, el horizonte siempre incierto, siempre confundiéndose con la tierra, entre celajes y vapores tenues, que no dejan, en la lejana perspectiva, señalar el punto en que el mundo acaba y principia el cielo" (25-26). La misma idea se encuentra en Tomás Eloy Martínez, para quien la extensión vasta de la pampa argentina y la escasez de ciudades incide de manera directa en la propensión de su país a las dictaduras.

2 Se conserva la ortograffa y la puntuación de la autora.

3 El motivo del aislamiento y la imposibilidad de salir debido a la condición isleña de Cuba ha encontrado repercusión en las obras de muchos escritores y poetas disidentes. Un ejemplo muy notable es el poema "La isla en peso" de Virgilio Piñera con su hidrofobia y el deseo de secar el mar como el único modo de recuperar la conexión perdida con el continente.

4 De modo semejante, De Ferrarri señala el "warlike trauma of loss (of dignity, of health, of humanity) that is the trademark of the Special Period" (194).

5 Ivette Gómez propone otra interpretación de las ruinas habaneras de Ponte: "La palabra ruina, cuya etimología proviene del verbo latino ruere que quiere decir 'caer' o 'derrumbarse,' suele asociarse con la caída de imperios. En este sentido, las ruinas habaneras suponen también la caída del 'imperio' soviético y el derrumbe de la relevancia de Cuba como una de sus más pintorescas provincias en el continente americano. Es el fin de la Guerra Fría el que precisamente coincide con el final de este imperio y con el inicio en la isla del 'Período Especial'”.

6 En correspondencia con Antonio José Ponte, en la cual expuse mi versión de una posible conexión entre su obra y la de Bulgakov, el autor me confirmó la 
existencia de un trasfondo bulgaquiano, aunque reconoció el carácter más intuitivo que literal de este: "Soy, desde hace muchos años, un lector admirado de El Maestro y Margarita, así como de otras obras de Bulgakov. He vuelto a leer esa novela hace poco, gracias a la aparición de una nueva traducción al español. Y he leído, además, las cartas de B. a Stalin, y lo publicado acerca de su expediente policial en los archivos de la KGB. Puede entonces que, tal como sugiere tu hipótesis, yo haya tenido presente a Bulgakov al dar mi testimonio en el documental de Borchmeyer. Y me parece muy atendible que en el fondo

de todo esto se encuentre la figura de Mefistófeles. Por supuesto, eso explicaría mucho en el urbanismo habanero: las promesas de bonanza que llevan a la destrucción han sido sostenidas tanto por Mefistófeles como por la propaganda del régimen revolucionario cubano. De manera que la coincidencia no es baladí. Sin embargo, tengo también que reconocer que un escritor no se conoce a fondo (de lo contrario no podría escribir nada) y que muchas veces son sus lectores quienes conocen de verdad lo que el escritor quiso escribir o significar. Me parece que en este caso es así, que sus hipótesis de lectura revelan algo que yo debo haber querido decir en ese entonces. Y, dada mi admiración por Bulgakov, me acojo gustosamente a las hipótesis que leo en su mensaje" (Ponte, "Desde la Universidad").

OBRAS CITADAS

A I A A. "Barbacoas y ruinas habaneras: fotografiar la miseria ajena”. Blogspot. Sentido Antropo-lógico. I8 Jan 2012. Web.

A P PADU RA I, AR Jun. Modernity At Large: Cultural Dimensions of Globalization. Minneapolis: U of Minnesota P, 1996.

BirkenMAIER, ANKE, y eSthER WhitFiELD. Introduction. Havana Beyond The Ruins: Cultural Mappings after 1989. Eds. Anke Birkenmaier y Esther Whitfield. Durham: Duke UP, 20II. I'I4.

в овеS, VEL I A CEC IL I A. "Visits to a Non-Place: Havana and Its Representation(s)". Havana Beyond The Ruins: Cultural Mappings after 1989. Eds. Anke Birkenmaier y Esther Whitfield. Durham: Duke UP, 20II. 15-31.

B U L G A ov, M IJ Aíl. El Maestro y Margarita. Trad. Amaya Lacasa Sancha. Madrid: Alianza Editorial, 1985.

C OYUla, maR I o. "The Bitter Trinquennium and the Dystopian City: Autopsy of a Utopia”. Havana Beyond The Ruins: Cultural Mappings after 1989. Eds. Anke Birkenmaier y Esther Whitfield. Durham: Duke UP, 20II. $3 \mathrm{I}^{-5}{ }^{2}$.

De ferrari, Guillermina. Community and Culture in Post-Soviet Cuba. New York:

Routledge, 20I4. 
Deleuze, gilles, and félix guattari. Anti-Oedipus: Capitalism and Schizophrenia. Trad. Robert Hurley, Mark Seem, y Helen R. Lane. New York: Viking Press, 1977.

DELGAD O, CARL OS. "Three Proposals Regarding Accelerated Urbanization Problems in Metropolitan Areas: The Lima Case". The American Behavioral Scientist I2.2 (1969): 34-45.

DEL REAL, PATRICIO, AND JoSEPH SCARPACi. "Barbacoas: Havana's New Inward Frontier". Havana Beyond The Ruins: Cultural Mappings after 1989. Eds. Anke Birkenmaier y Esther Whitfield. Durham: Duke UP, $2011.53^{-} 72$.

F I GES, ORLA N D. The Whisperers: Private Life in Stalin's Russia. New York: Metropolitan Books, 2007.

G ó m E Z, IV t T te. "Simulaciones de la memoria: Antonio José Ponte y Tuguria, la ciudad-ruina". La Habana Elegante 47 (2010): S. pag. Web.

Habana - Arte nuevo de hacer ruinas. Dir. Florian Borchmeyer. Raros Media, 2006. H A RE Y, DA Vid. The Condition of Postmodernity. Cambridge: Blackwell, I990. -. "The Urbanization of Anti-Capitalist Struggle". Annual Humanities Lecture. Boston University, Photonics Center, Boston. I8 Oct. 20I2. Lecture.

H OSEK, JENnifer RUth. "Transnational Cinema and the Ruins of Berlin and Havana: Die neue Kunst, Ruinen zu bauen (The New Art of Making Ruins, 2007) and Suite Habana (2003)". Spatial Turns: Space, Place, and Mobility in German Literary and Visual Culture. Eds. Jaimey Fisher y Barbara Caroline Mennel. New York: Rodopi, 20IO. I89-2II.

LAWless, CEC Ela. "Urban Performance Pieces in Fragmented Form: A Reading of Pedro Juan Gutiérrez and Antonio José Ponte". Havana Beyond The Ruins: Cultural Mappings after 1989 . Eds. Anke Birkenmaier y Esther Whitfield. Durham: Duke UP, 20II. I87-208.

MARTínEZ, TOMÁS ELOY. "Mito, historia y ficción en América Latina”. Encuentros 32 (I999): I-I4.

MATVEEV, YEVGENi. "Nezhiloye v zhilom:" prostranstvo domov "novogo tipa" v SSSR v kontekste sotsialnyh izmenenii kontsa $1950-\mathrm{h}$ nachala $1960-\mathrm{h}$ gg". Vestnik molodyh uchenyh PGNIU. 3 (2013): 254-258.

MONREAL GONZÁLEZ, PEDRO. "Las redes productivas globales y los límites de las estrategias 'auto-centradas' de desarrollo: el caso de Cuba durante la década

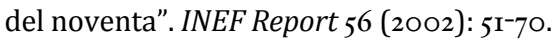

P ONTE, Antonio josé. "Desde la Universidad de Toronto." Mensaje a la autora. 23 abril 20I5. E-mail.

-. "La Habana: City and Archive". Havana Beyond The Ruins: Cultural Mappings after 1989. Eds. Anke Birkenmaier y Esther Whitfield. Durham: Duke UP, 20II. 249269. 
-. "La Habana: Un paréntesis de ruinas en Cuba". Counterpoints on Culture, History, and Society. Eds. Francisco A. Scarano y Margarita Zamora. San Juan: Ediciones Callejón, 2007. 6I-90.

-. "Un arte de hacer ruinas." Un arte de hacer ruinas y otros cuentos. México D.F.: Fondo de Cultura Económica, 2005: 23-40.

-. Villa Marista en plata. Madrid: Colibri, 2010.

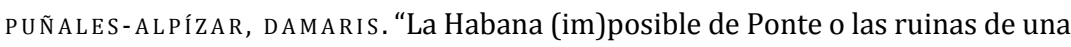
ciudad atravesada por una guerra que nunca tuvo lugar". Ciberletras 20 (2008): S. pag. Web.

REMBOLD, ELFIE, y PETER CARRIER. "Space and Identity: Construction of National Identities in an Age of Globalisation". National Identities 4.I3 (20II): 36I-377.

RODRÍGUEZ, NÉSTOR. "La mirada epistemológica en la poética literaria de Antonio José Ponte". Revista Canadiense de Estudios Hispánicos 33.3 (2009): 566-577.

RoJAS, RAFAE L. "The Illegible City: Havana after the Messiah". Havana Beyond The Ruins: Cultural Mappings after 1989. Eds. Anke Birkenmaier y Esther Whitfield. Durham: Duke UP 20II. II9-I34.

Sarmiento, domingo faustino. Facundo. Buenos Aires: La Facultad, i92I.

SERnA, MERCEDES, y anna Solana. "Entrevista a Antonio José Ponte". Cuadernos hispanoamericanos 665 (2005): 127-134.

S I M M E L, G E O R G. "Las ruinas". Sobre la aventura. Ensayos filosóficos. Trad. Gustau Muñoz y Salvador Mas. Barcelona: Ediciones Península, I988. I8I-193.

SiMM E L, GEOR G. "Venice". Theory, Culture \& Society 24 (2007): 42-46.

T UROMA, SAnNA. "Semiotika gorodskogo prostranstva Yu.M. Lotmana: opyt pereosmysleniia." Novoe literaturnoe obozrenie 98 (2009):66-76. 